\title{
Sorge um die Qualität der Aus- und Weiterbildung
}

\author{
Die Schweizer Hausärzte setzen sich ein für die Qualität der künftigen Aus- und \\ Weiterbildung und intervenieren bei den zuständigen Instanzen. Nachfolgend \\ veröffentlicht die Schweizerische Ärztezeitung einen Brief von KHM, SGAM, \\ SGIM und SGP an die Adresse des Präsidenten der Schweizerischen Medizi- \\ nischen Interfakultätskommission (SMIFK), Prof. Charles Bader.
}

Grindelwald/ Genf/Luzern, 25. Juni 2004

Sehr geehrter Herr Professor

Aus wachsender Sorge um die Qualität der Ausund Weiterbildung der künftigen Ärztinnen und Ärzte gelangen wir, die Organisationen der Schweizer Hausärztinnen und Hausärzte, heute an die Schweizerische Medizinische Interfakultätskommission SMIFK.

Wir beobachten mit zunehmendem Unbehagen, wie in letzter Zeit die von den Medizinischen Fakultäten der Schweizer Universitäten über viele Jahre mit grossem Einsatz aufgebauten Reformprogramme der universitären Ausbildung der Medizinalberufe und der dafür in breitem Konsens geschaffene Entwurf für ein neues Medizinalberufegesetz durch unkoordinierte und sogar konkurrierende Aktionen von Gruppen und Institutionen mit bildungspolitischer Ausrichtung (Vorschlag der Arbeitsgruppe Kleiber, Gegenvorschlag der CRUS, Bologna-Richtlinien, Akkreditierungsprozeduren Weiterbildung) verzögert und blockiert werden. Die daraus entstandenen struktur- und ressourcenbezogenen Interessenkonflikte blenden nach unseren Beobachtungen und Erfahrungen die zentrale Bedeutung der Medizinalberufeausbildung für die Gesundheitsversorgung der Schweizer Bevölkerung aus. Damit vernachlässigen sie den klaren gesellschaftlichen Auftrag an die ärztliche Ausund Weiterbildung und setzen die Qualität der Gesundheitsversorgung aufs Spiel.

Dies ist für die Organisationen der Schweizer Hausärztinnen und Hausärzte nicht länger annehmbar, denn wir tragen heute und in Zukunft die Verantwortung für einen wesentlichen Teil der gesundheitlichen Versorgung der Schweizer Bevölkerung.

Das Wohl der Patienten, der Auftrag der Bevölkerung an die Gesundheitsversorgung wie auch die Wissenschaftlichkeit der Bildung verlangen, dass
- die medizinische Aus-, Weiter- und Fortbildung aller Ärztinnen und Ärzte auf eine optimale Qualität der medizinischen Versorgung ausgerichtet werden;

- die Phasen der Aus-, Weiter- und Fortbildung als in sich zusammenhängendes Kontinuum verstanden und akzeptiert werden und

- die Ziele der Aus-, Weiter- und Fortbildung dementsprechend formuliert, respektiert und umgesetzt werden, wobei die wissenschaftlichen und die berufsbezogenen Kompetenzen kohärent aufeinander abzustimmen sind.

Die medizinischen Fakultäten haben diese der Medizinalberufereform zugrundeliegenden Kriterien sowohl mitgeprägt als auch in ihre Reformprogramme aufgenommen und damit eine Führungsrolle übernommen. Auch bei der medizinischen Weiterbildung und der lebenslangen Fortbildung der Medizinalberufe spielen sie eine wichtige Rolle. Die Schweizer Organisationen der Hausärztinnen und Hausärzte haben sie dabei mit allen Kräften und Möglichkeiten unterstützt.

Im Interesse der übergreifenden gemeinsamen Aufgabe und im übergeordneten Interesse der Gesundheit von Bevölkerung und Individuum möchten wir diese Zusammenarbeit mit der SMIFK und den medizinischen Fakultäten noch verstärken. Wir bieten Ihnen unsere politische, inhaltliche und personelle Unterstützung an, um insbesondere drei wichtige Schritte unverzüglich an die Hand zu nehmen:

1. die zügige Inkraftsetzung des Medizinalberufegesetzes (MedBG) als gesetzliche Grundlage für die bereits laufenden Reformprogramme;

2. die Operationalisierung der allgemeinen und speziellen Lernziele des Schweizer Lernzielkatalogs;

3. die kompetenzorientierte Gestaltung des Kontinuums von ärztlicher Aus- und Weiterbildung.

Wir bitten die SMIFK um Stellungnahme und grüssen Sie freundlich 
Namens der Organisationen der Schweizer Hausärztinnen und Hausärzte

Dr. med. Marc Müller

Präsident Kollegium für Hausarztmedizin KHM

Prof. Dr. med. Verena Briner

Präsidentin Schweizerische Gesellschaft für Innere Medizin SGIM

Dr. med. Jacques de Haller

Präsident Schweizerische Gesellschaft für Allgemeinmedizin SGAM

Dr. med. Pierre Klauser

Präsident Schweizerische Gesellschaft für Pädiatrie SGP

\section{Kopie an}

- Bundesrat Pascal Couchepin, Chef des Eidg. Departements des Inneren EDI;

- Staatssekretär Charles Kleiber, Gruppe für Wissenschaft und Forschung GWF;

- GDK Schweizerische Konferenz der kantonalen Gesundheitsdirektorinnen und -direktoren;

- CRUS Rektorenkonferenz der Schweizer Universitäten;

- SGK Kommissionen für soziale Sicherheit und Gesundheit von National- und Ständerat;

- Prof. Thomas Zeltner, Direktor des Bundesamts für Gesundheit;

- Leitenden Ausschuss für die eidgenössischen Medizinalprüfungen;

- Eidg. Weiterbildungsausschuss für medizinische Berufe;

- Zentralvorstand der Verbindung der Schweizer Ärztinnen und Ärzte FMH.

\section{Inquiétude concernant la qualité de la formation prégraduée et postgraduée}

Les médecins de premier recours suisses s'engagent pour la qualité de la formation médicale de l'avenir et interviennent auprès des instances concernées. Le Bulletin des médecins suisses publie ci-dessous une lettre du CMPR, de la SSMG, de la SSMI et de la SSP à I'adresse du Président de la Commission interfacultaire médicale suisse, $M$. le professeur Charles Bader.

Grindelwald/Genève/Lucerne, 25 juin 2004

Monsieur le Professeur,

Les soussignés, représentant les organisations des médecins de premier recours de Suisse, s'adressent aujourd'hui à la Commission interfacultés médicale suisse (CIMS) en raison d'une inquiétude croissante concernant la qualité de la formation des futurs médecins, tant au niveau prégradué que postgradué.

Plusieurs initiatives concurrentes et incoordonnées de divers groupes et instances en rapport avec la politique de la formation (propositions du groupe Kleiber, contre-propositions de la CRUS, directives de Bologne, procédures d'accréditation des programmes de formation postgraduée) nous semblent mettre en péril la poursuite du processus de réforme du curriculum des études de médecine, à laquelle les facultés suisses travaillent avec beaucoup d'engagement depuis plusieurs années, ainsi que la mise en application d'une nouvelle loi sur les professions médicales qui est le fruit d'un large consensus.

Les conflits d'intérêt à propos de la structure responsable de cette formation ainsi qu'à propos des ressources nécessaires, nous semblent mettre de côté l'importance centrale de la formation des professions médicales pour la dispensation des soins de santé à la population suisse.

Les instances responsables de la formation médicale tant pré- que postgraduée ont une mission sociale claire et l'oublier risque de mettre en danger la qualité des soins à la population.

Cette situation est inacceptable pour les organisations des médecins de premier recours suisses, représentant les fournisseurs de la majeure partie des soins de santé à la population suisse, tant aujourd'hui que demain.

Le bien-être du patient, le droit de la population à des soins de santé de qualité ainsi que la qualité scientifique de la formation des médecins exigent que: 
- la formation médicale (tant prégraduée que postgraduée et continue) soit orientée vers des soins de santé de qualité optimale;

- les différentes étapes de la formation médicale, c'est à dire la formation prégraduée, postgraduée et continue, soient comprises et acceptées comme faisant partie d'un continuum cohérent;

- les objectifs de la formation prégraduée, postgraduée et continue soient explicitement formulés, respectés, et appliqués en tenant compte tant de compétences scientifiques que de compétences professionnelles.

Les facultés de médecine de Suisse ont pris un rôle de meneur dans cette réforme des professions médicales en définissant les critères à la base de cette réforme et en les intégrant dans leur curriculum. Ces facultés jouent aussi un rôle central dans la formation prégraduée, postgraduée et continue de toutes les professions médicales. Les organisations des médecins de premier recours suisses ont soutenu avec ardeur ce travail des facultés.

Dans l'intérêt supérieur des soins de santé à la population et aux individus, une action globale s'impose, avec un renforcement de la collaboration avec la CIMS et les facultés de médecine.

Nous proposons notre soutien tant politique qu'opérationnel aux trois actions suivantes:

1. entrée en vigueur rapide de la loi sur les professions médicales (LPMed) comme base législative des programmes de réforme déjà en cours;

2. opérationnalisation des objectifs pédagogiques généraux et spécifiques du Catalogue suisse des objectifs d'apprentissage pour la formation médicale prégraduée;

3. une conception du continuum de la formation médicale pré- et postgraduée basée sur les compétences.
Nous demandons à la Commission interfacultés médicale suisse de prendre position et vous adressons nos meilleures salutations.

$\mathrm{Au}$ nom des organisations des médecins de premier recours

\section{Dr Marc Müller}

Président du Collège suisse des médecins de premier recours CMPR.

\section{Professeur Verena Briner}

Présidente de la Société suisse de médecine interne SSMI

\section{Dr Jacques de Haller}

Président de la Société suisse de médecine générale SSMG

\section{Dr Pierre Klauser}

Président de la Société suisse de pédiatrie SSP

\section{Copie}

- Monsieur le Conseiller fédéral Pascal Couchepin, chef du Département fédéral de l'intérieur

- Monsieur le Secrétaire d'Etat Charles Kleiber, Groupement de la science et de la recherche

- Conférence suisse des directrices et directeurs cantonaux de la santé

- Conférence des recteurs des universités suisses

- Commission parlementaire de la sécurité sociale et de la santé publique

- Monsieur le Professeur Thomas Zeltner, directeur de l'Office fédéral de la santé publique

- Comité directeur pour les examens des professions médicales

- Comité de la formation postgrade pour les professions médicales

- Comité central de la Fédération des médecins suisses 